\title{
Lactobacilles, oxygène, métabolisme et antagonisme
}

\author{
L Frey, JC Hubert \\ Laboratoire de microbiologie, Université Louis-Pasteur, URA1481 CNRS, \\ Institut Le-Bel, 4, rue Blaise-Pascal, 67070 Strasbourg, France
}

\begin{abstract}
Résumé - Les lactobacilles homo- et hétérofermentaires facultatifs oxydent le glucose; le pyruvate formé est normalement réduit en lactate par la lactate deshydrogénase (LDH) et s'accumule dans la plupart des cas. La LDH de certaines de ces bactéries est activée par le fructose 1,6 diphosphate (FDP). En l'absence de ce dernier, la LDH a une activité faible et le catabolisme aboutit à des produits terminaux tels l'acétate l'acétoïne ou l'éthanol. Chez d'autres lactobacilles en particulier chez Lactobacillus plantarum qui présentent une LDH FDP indépendante on constate qu'en présence d'oxygène après épuisement du glucose du milieu le lactate est métabolisé et transformé en acétate. La régulation des mécanismes n'est pas connue. Les différents systèmes enzymatiques pouvant être envisagés pour expliquer cette transformation ont été pris en considération : lactate deshydrogénase NAD indépendante, lactate oxydase, pyruvate formate lyase, pyruvate deshydrogénase, pyruvate oxydase ainsi que NADH oxydase, avec production de peroxyde d'hydrogène ou d'eau ou encore NADH peroxydase. Ces activités ont été testées chez $L$ plantarum et chez Lactobacillus sake cultivés dans les mêmes conditions. Nous avons pu mettre en évidence une pyruvate oxydase chez $L$ plantarum uniquement en culture aérobie. Cette potentialité de production d'acétate à partir de lactate chez certaines souches est la raison de leur activité antagoniste à l'égard de la croissance des pseudomonades en cultures mixtes.
\end{abstract}

\section{métabolisme / oxygène / lactobacilles / antagonisme / Pseudomonas}

Summary - Lactobacilli, oxygen, metabolism and antagonism. During the glucose oxidation by the homo- and hetero-fermentative Lactobacilli, the pyruvate which is formed is subsequently reduced by the lactate dehydrogenase ( $L D H)$ into lactate which accumulates in the cell. In some bacteria, the $L D H$ is activated by fructose 1,6 diphosphate (FDP). When the FDP is absent, the LDH activity is low and the glucose catabolism leads to terminal products such as acetate, acetoin or ethanol. In another Lactobacillus such as Lactobacillus plantarum, the LDH is FDP-independent but lactate is metabolized into acetate in the presence of oxygen and glucose depletion. The regulation of this process is unknown. Several enzymes may be involved in this process; the NAD-independent lactate dehydrogenase; the lactate oxidase; the pyruvate formate lyase; the pyruvate dehydrogenase; the pyruvate oxidase and the $\mathrm{NADH}$ oxidase with formation of $\mathrm{H}_{2} \mathrm{O}_{2}$ or $\mathrm{H}_{2} \mathrm{O}$ and also the $\mathrm{NADH}$ peroxidase. These activities were tested in $\mathrm{L}$ plantarum and $\mathrm{L}$ sake grown in similar conditions. A pyruvate oxidase activity was detected only in $\mathrm{L}$ plantarum in aerobiosis. We have shown that the acetate produced from lactate inhibits the growth of pseudomonads in oxygenated cocultures. 


\section{INTRODUCTION}

Les bactéries lactiques produisent à partir des sucres de l'acide lactique dans le cas des bactéries homofermentatives ou de l'acide lactique, de l'éthanol, du $\mathrm{CO}_{2}$ de l'acétate dans le cas des hétérofermentatives. Ces produits terminaux s'accumulent dans les milieux de culture. Des changements de métabolisme avec passage d'un système homofermentaire à un bilan héterofermentaire peuvent être observés dans certains cas, mettant en jeu des régulations d'activités enzymatiques nombreuses. Ces déviations, souvent fonction de l'environnement biochimique ou physicochimique au cours de la croissance, sont d'importance fondamentale dans le contrôle des procédés mettant en cuuvre ces micro-organismes soit en culture pure soit en culture mixte dans des milieux naturels. Notre intérêt s'est porté sur Lactobacillus plantarum, une espèce homofermentative largement utilisée comme levain pour l'élaboration de produits fermentés, dans le domaine agroalimentaire en particulier. Quelques représentants de cette espèce présentent la propriété d'inhiber la croissance des pseudomonades en culture mixte. Sachant que ces bactéries sont la plupart du temps présentes dans les mêmes milieux que les bactéries lactiques, qu'elles sont responsables dans de nombreux cas de déviation du procédé de fermentation vers une forte protéolyse alcaline accompagnée d'une putréfaction, nous avons essayé d'analyser les mécanismes qui sous-tendent la potentialité d'arrêter la croissance des Pseudomonades.

\section{MATÉRIEL ET MÉTHODES}

\section{Milieux et conditions de culture}

Les cultures mixtes ont été réalisées sur milieu complexe au jus de tomate (Bernstein et Wood,
1957). Les bactéries lactiques sont cultivées également sur milieu MRS (de Man et al, 1960) et $P$ fluorescens sur bouillon ordinaire. Les cultures en bioréacteur de 21 sont faites à $25^{\circ} \mathrm{C}$, avec régulation du $\mathrm{pH}$ à 5,8 avec régulation de la $\mathrm{PO}_{2}$ à $25 \%$ de saturation.

\section{Organismes utilisés}

L plantarum CCM 1904; Lactobacillus sake 207 provient de la collection du $\mathrm{Dr} J$ Fournaud (INRA Jouy en Josas) et a été identifié par le $\mathrm{Dr}$ M-C Montel et al (1991) ; Pseudomonas fluorescens CCM 2799.

\section{Analyses du milieu de fermentation}

Le glucose est déterminé par la méthode à I'hexokinase et glucose 6-P deshydrogénase Kit Boehringer. Le lactate et l'acétate sont déterminés par CLHP système Waters (Millipore SA) avec une colonne supelco $\mathrm{C} 610 \mathrm{H}$, la phase mobile est l'acide phosphorique $0,1 \%$, le débit est réglé à $0,5 \mathrm{ml} / \mathrm{min}$, l'élution est faite sur 30 min et la détection en UV à $210 \mathrm{~nm}$; la quantification des acides organiques est faite grâce au programme Baseline 810 de Waters.

\section{Déterminations enzymatiques}

Préparation des extraits cellulaires: les bactéries récoltées et lavées sont mises en suspension dans le tampon phosphate de $\mathrm{Na} 0,1 \mathrm{~mol} / \mathrm{l} \mathrm{pH}$ 5,8 . Le broyage se fait avec un vibrobroyeur Retsch MM2 avec des billes de $0,25 \mathrm{~mm}$ pendant $10 \mathrm{~min}$. Aprés broyage, les tubes sont centrifugés $10 \mathrm{~min}$ à $12000 \mathrm{~g}$ à froid; le surnageant clair est utilisé immédiatement pour les déterminations enzymatiques.

Les activités de la NADH oxydase, de la pyruvate oxydase et de la lactate oxydase sont déterminées par une méthode polarographique avec une microélectrode de Clark. L'activité spécifique des trois enzymes est exprimée en nmol $\mathrm{d}^{\prime} \mathrm{O}_{2}$ absorbé par min et par mg de protéine. L'activité de la lactate deshydrogénase NAD indépendante est déterminée d'après la méthode de Snoswell (1966). 


\section{Révélation de la pyruvate oxydase}

Les protéines sont séparées par électrophorèse sur gel de polyacrylamide à $10 \%$ en présence de SDS (sodium dodécylsulfate). Les anticorps antipyruvate oxydase sont obtenus par injection à des lapins de pyruvate oxydase de Pediococcus (Sigma). La révélation par immunodétection est faite par la technique ELISA (Engvall et Perlmann, 1971).

\section{RESULTATS}

\section{Mise en évidence de l'interaction de type amensalistique (0-) entre lactobacilles et pseudomonades}

Beaucoup d'études faites dans des conditions de cultures plus ou moins contrôlées mettent en évidence des inhibitions de croissance sans toutefois pouvoir donner de façon définitive les raisons de l'interaction. Suivant les recherches, sont incriminées: des bactériocines, des produits du métabolisme tels que des acides organiques, le $\mathrm{pH}$ ou le peroxyde d'hydrogène. Nous avons réalisé des cultures mixtes bactéries lactiques-pseudomonades en bioréacteur (LSL Biolaffitte) de 2 I de capacité, avec régulations de température, de $\mathrm{pH}$ et de $\mathrm{pO}_{2}$. La figure 1 rassemble les courbes de croissance obtenues sur le même milieu dans les conditions standard.

La souche test de $P$ fluorescens est cultivée seule ou en présence de $L$ plantarum CCM1904 ou de $L$ sake 207 . Un arrêt de la croissance, puis une diminution du nombre de cellules de Pseudomonas pouvant former colonie est visible dans le cas du couple $L$ plantarum- $P$ fluorescens. D'autres essais nous montrent que la présence de Pseudomonas n'affecte en rien la croissance de $L$ plantarum. L sake 207 comme d'autres lactobacilles testés n'a aucune influence sur la culture de Pseudomonas (neutralisme ou interaction de type 0-0).

\section{Caractérisation du mécanisme de l'inhibition}

\section{Mise en évidence d'une formation d'acétate}

Le fractionnement du milieu de culture résultant soit de la culture mixte $L$ plantarum-

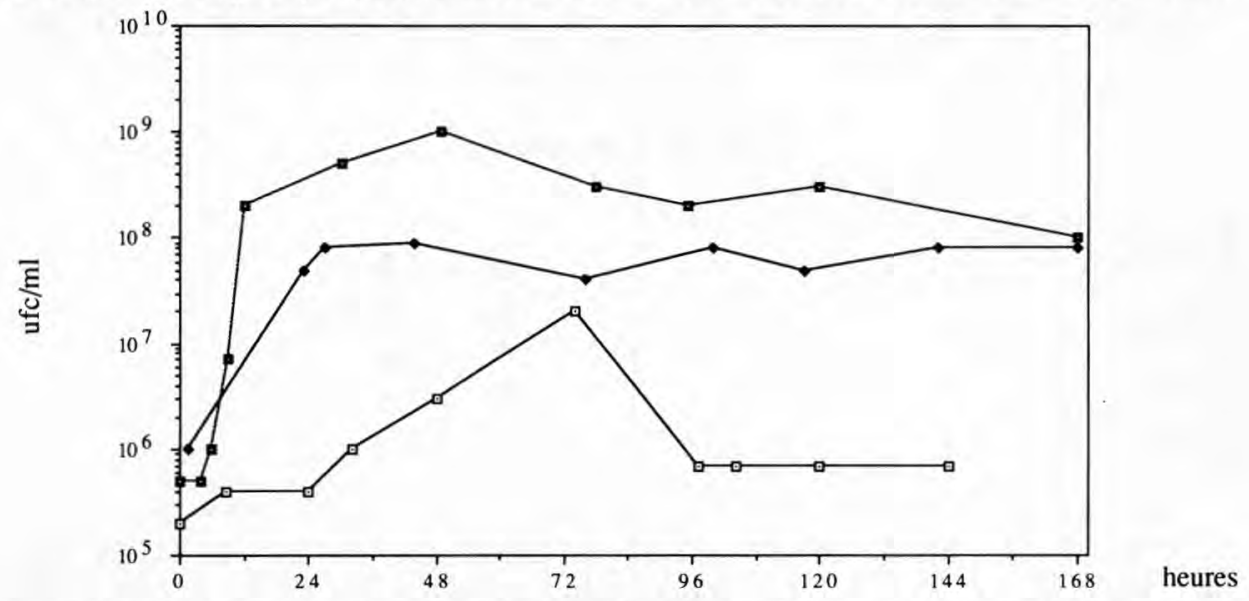

Fig 1. Croissance de $P$ fluorescens seul et en culture mixte. $P$ fluorescens; $\square P$ fluorescens et $L$ plantarum; $\bullet P$ fluorescens et $L$ sake 207. 
$P$ fluorescens soit de la culture pure de $L$ plantarum et comparé au fractionnement du milieu de culture résultant des expériences avec $L$ sake pris comme témoin, révèle la présence d'acétate dans les mi- lieux avec $L$ plantarum. La figure 2, qui représente les dosages par CLHP, en fonction de la durée de la culture révèle que l'acétate apparaît après disparition du glucose et aux dépens du lactate dans le cas

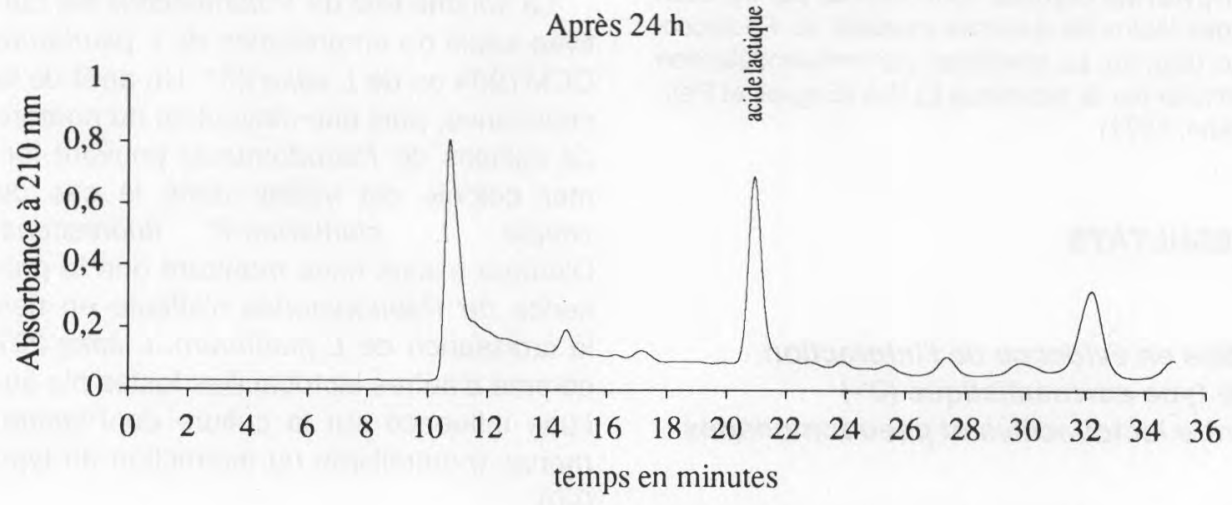

Après $46 \mathrm{~h}$

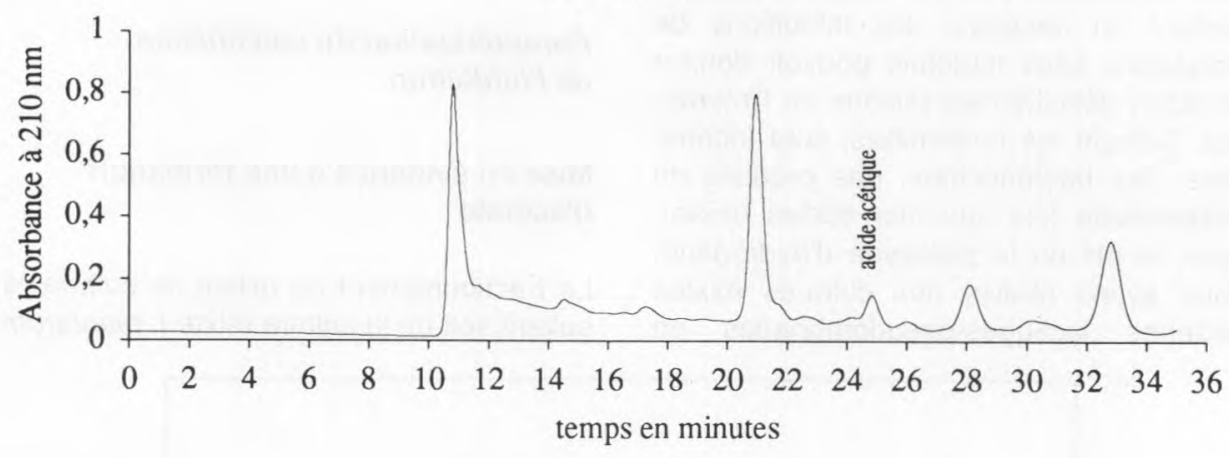

Après $101 \mathrm{~h}$ de culture

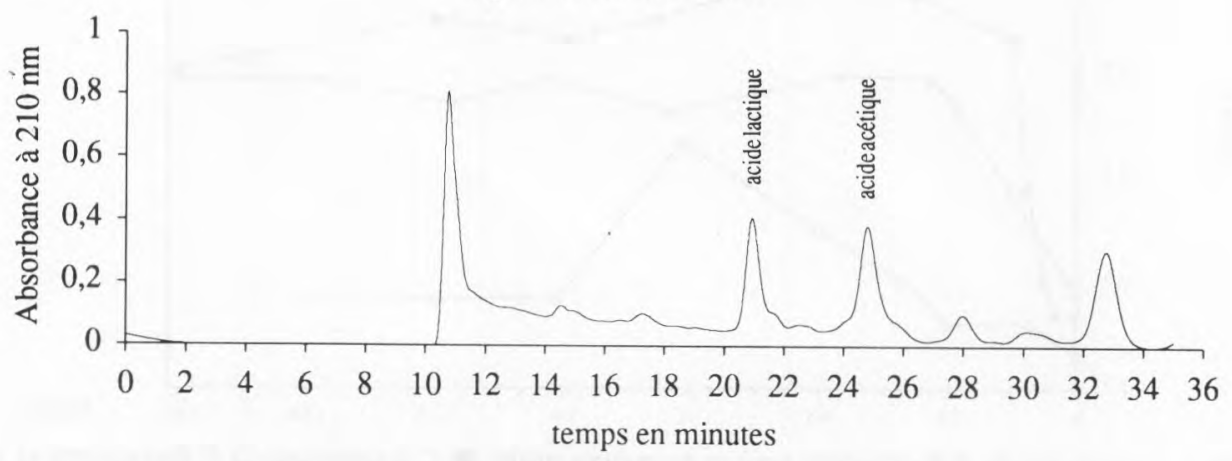

Fig 2. Évolution de la production d'acides dans une culture de $L$ plantarum CCM 1904. 
de $L$ plantarum. La figure 3 présente les mêmes dosages avec $L$ sake. Chez cette souche, le lactate s'accumule.

Dans des conditions d'anaérobiose stricte (bioréacteur maintenu en continu sous courant d'azote), le lactate s'accumule dans la culture de $L$ plantarum et n'est jamais converti en acétate. L'oxygène joue donc un rôle essentiel dans la régulation métabolique qui s'opère chez $L$ plantarum.

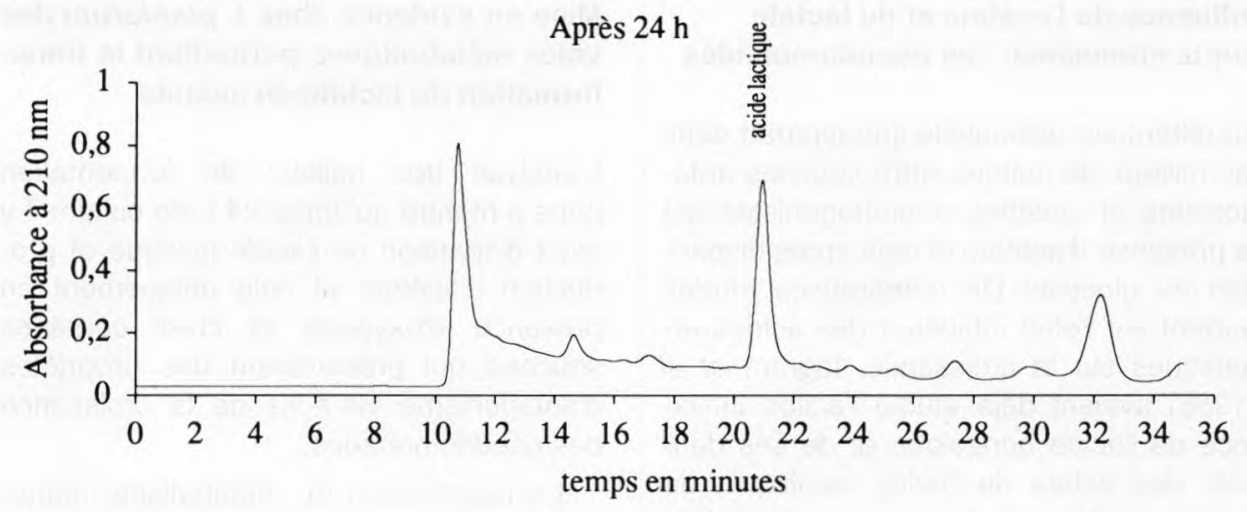

Après $48 \mathrm{~h}$
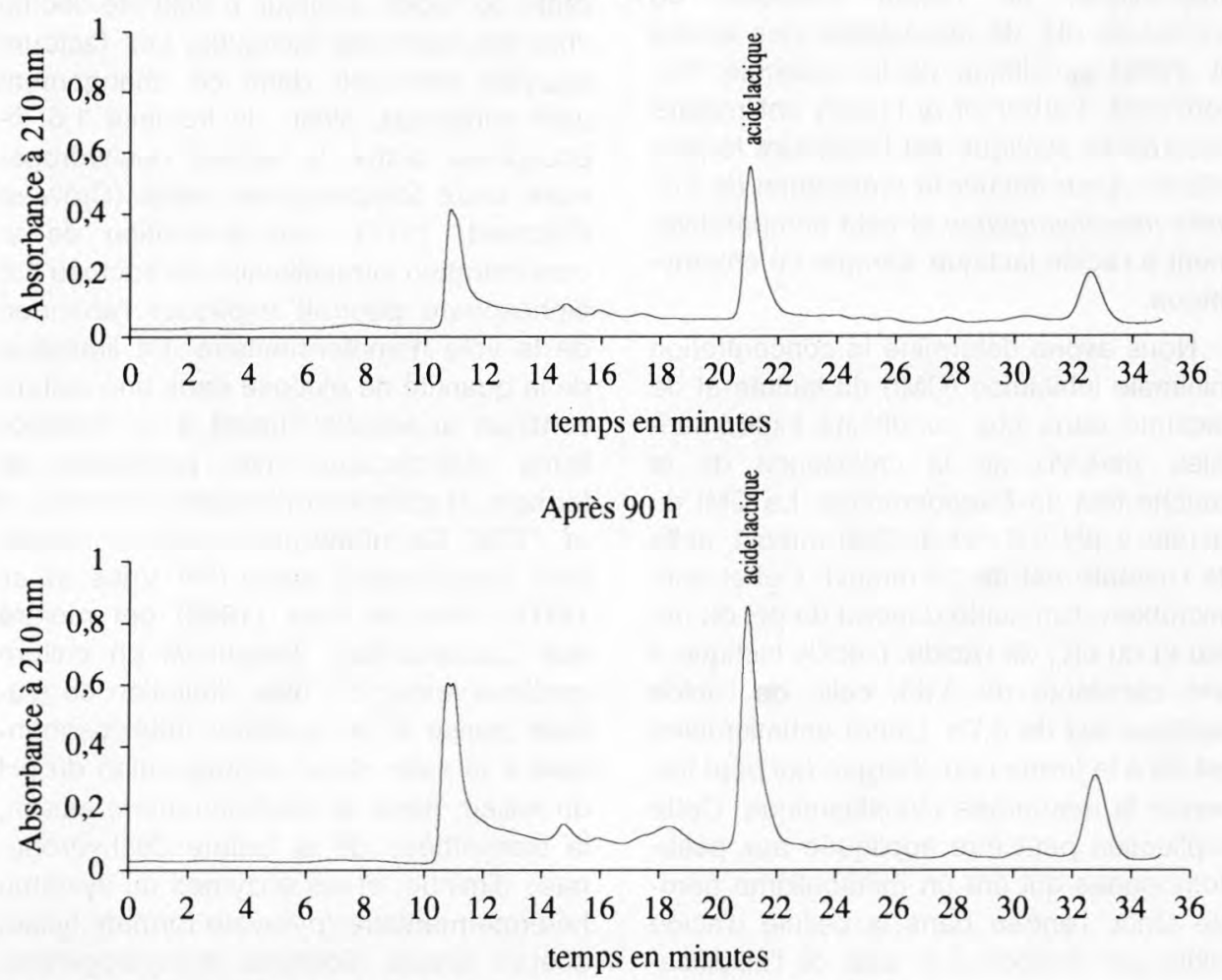

Fig 3. Évolution de la production d'acides dans une culture de L sake 207 . 
Aucune bactériocine de nature protéique ou peptidique n'a pu être mise en évidence, par fractionnement dans ces mêmes milieux.

\section{Influence de l'acétate et du lactate sur la croissance des pseudomonades}

La différence essentielle qui apparaît dans les milieux de culture entre souches antagonistes et souches nonantagonistes est la présence d'acétate et cela après disparition du glucose. De nombreuses études portent sur l'effet inhibiteur des acides organiques sur la croissance. Ingram et al (1956) avaient déjà étudié l'action inhibitrice de l'acide benzoique et de ses dérivés, des esters de l'acide vanillique, de l'acide acétique et de ses sels, de l'acide propionique, de l'acide sorbique, en termes de $\mathrm{pH}$, de dissociation des acides et d'effet spécifique de la molécule. Récemment, Farber et al (1989) ont montré que l'acide acétique est l'acidifiant le plus efficace pour inhiber la croissance de Listeria monocytogenes et cela comparativement à l'acide lactique, citrique ou chlorhydrique.

Nous avons déterminé la concentration minimale inhibitrice (CMI) du lactate et de l'acétate dans nos conditions expérimentales vis-à-vis de la croissance de la souche test de Pseudomonas. La CMI du lactate à $\mathrm{pH} 5,8$ est de $200 \mathrm{mmol} /$, celle de l'acétate est de $20 \mathrm{mmol} / \mathrm{l}$. L'effet antimicrobien d'un acide dépend du $\mathrm{pH}$ du milieu et du $\mathrm{pK}_{\mathrm{a}}$ de l'acide. L'acide lactique a une constante de 3,86 , celle de l'acide acétique est de 4,75. L'effet antimicrobien est dû à la forme non chargée qui peut traverser la membrane cytoplasmique. Cette explication peut être appliquée aux pseudomonades qui ont un métabolisme aérobie strict; l'entrée dans la cellule d'acide faible non dissocié à la suite de l'acidification du milieu extérieur oblige la cellule à rétablir son $\mathrm{pH}$ interne. Les mécanismes régulateurs utilisent l'énergie chimioosmotique jusqu'à épuisement et conduisent à l'arrêt de la croissance.

\section{Mise en évidence chez $L$ plantarum des voies métaboliques permettant la trans- formation du lactate en acétate}

L'analyse des milieux de fermentation nous a montré qu'après $24 \mathrm{~h}$ de culture il y avait disparition de l'acide lactique et production d'acétate et cela uniquement en présence d'oxygène et chez certaines souches qui présentaient des propriétes d'antagonisme vis-à-vis de la croissance des pseudomonades.

La modification du métabolisme homofermentaire vers un bilan laissant apparaitre de l'acide acétique a déjà été décrite chez les bactéries lactiques. Les facteurs pouvant intervenir dans ce changement sont nombreux, ainsi : le fructose 1,6 diphosphate active la lactate deshydrogénase chez Streptococcus lactis (Crow et Pritchard, 1977); une diminution de la concentration intracellulaire de fructose 1,6 diphosphate pourrait expliquer l'abandon de la voie homofermentaire. La limitation de la quantité de glucose dans une culture continue anaérobie aboutit à un métabolisme hétérolactique avec production de formate, d'acétate et d'éthanol (Thomas et al, 1979). Ce même phénomène est décrit pour Lactobacillus casei (De Vries et al, 1970). Rhee et Park (1980) ont montré que Lactobacillus bulgaricus en culture continue anaérobie avec limitation de glucose passe à un système hétérofermentaire à la suite d'une augmentation du $\mathrm{pH}$ du milieu; dans un environnement alcalin, la biosynthèse de la lactate deshydrogénase diminue, et les enzymes du système hétérofermentaire (pyruvate formate lyase, acétate kinase, aldéhyde deshydrogénase et phosphotransacétylase) sont activés. 
Lloyd et al (1978), Hickey et al (1983) montrent que l'oxydation du pyruvate en acétate est possible chez différents lactobacilli ( $L$ bulgaricus, $L$ casei, $L$ lactis, $L$ delbrueckii, $L$ acidophilus et $L$ plantarum). Thomas (1987) propose deux mécanismes pour la formation d'acétate dans les fromages; l'un oxygène dépendant avec comme substrat le lactate, l'autre oxygène indépendant avec comme substrat le citrate. Tseng et Montville (1990) constatent des changements d'activités enzymatiques dans des cultures aérobies comparativement aux cultures anaérobies, ainsi que des effets en relation avec des variations de $\mathrm{pH}$. II ressort de toutes ces études l'énorme potentialité métabolique que détiennent ces bactéries capables de modifier leurs voies métaboliques fermentaires principales en fonction de l'environnement nutritionnel ou physico-chimique.

Dans le cas des cultures mixtes $L$ plantarum- $P$ fluorescens nous avons essayé de mettre en évidence les systèmes enzymatiques capables de rendre compte de la transformation en aérobiose du lactate en acétate.

\section{Activités enzymatiques pouvant être impliquées dans la transformation du lactate en acétate}

On peut envisager soit un passage direct, soit un passage par l'intermédiaire du pyruvate.

Pour la transformation lactate ---> pyruvate, plusieurs systèmes enzymatiques sont décrits soit chez des bactéries lactiques, soit pour L plantarurn:

- avec intervention de la lactate déshydrogénase NAD indépendante : Strittmatter (1959) montre que $L$ plantarum oxyde le lactate en pyruvate en présence d'accepteur d'électrons artificiels tels que le bleu de méthylène ou le dichlorophénolindophénol;
- par la lactate oxydase : London (1968, 1990) montre la présence chez Streptococcus faecium d'une lactate oxydase comprenant une flavine et utilisant l'oxygène comme accepteur d'électrons avec production de peroxyde d'hydrogène; une lactate oxydase particulière (lactate-2 monooxygénase décarboxylase) a été obtenue purifiée à partir de Mycobacterium smegmatis par Sullivan (1968), et son mécanisme d'action précisé par Lockridge et al (1972). Ce système enzymatique conduit chez cette bactérie à l'oxydation directe du lactate et à sa décarboxylation sans passage par l'étape pyruvate, soit :

$$
\text { lactate }+\mathrm{O}_{2} \rightarrow \text { acétate }+\mathrm{CO}_{2}+\mathrm{H}_{2} \mathrm{O}_{2}
$$

Une activité lactate oxydase a été mise en évidence chez différents lactobacilles, dont Lactobacillus curvatus, Lactobacillus sake, Lactobacillus bulgaricus par Kandler (1983). L'existence d'une telle protéine enzymatique, lactate oxydase spécifique pour L plantarum n'est pas prouvée ; il pourrait s'agir simplement d'une activité de deshydrogénation suivie d'une NADH oxydation et ou d'une pyruvate oxydation.

II reste donc beaucoup d'imprécisions concernant le mode d'action enzymatique au niveau de l'oxydation directe du lactate chez les bactéries lactiques en général. De plus, la détermination des activités est toujours faite sur des extraits et ne peut exclure la participation de la lactate deshydrogénase et de la pyruvate oxydase comme le soulignent Murphy et al (1985). Chez $L$ plantarum, la lactate deshydrogénase NAD dépendante fonctionne essentiellement en faveur de la production de lactate.

En ce qui concerne la transformation pyruvate $\rightarrow->$ acétate, à partir du pyruvate 3 voies sont possibles :

- par la pyruvate formate lyase : le pyruvate est transformé en formate et en acétyl 
CoA par la pyruvate formate lyase en anaérobiose uniquement; cette activité a été trouvée chez les streptocoques par Thomas et al (1979) et Abbe et al (1982) et plus récemment pour $L$ plantarum par Lindgren et al (1990); l'activité est induite par le citrate; mais cette activité ne peut être prise en compte dans notre cas (aérobiose);

- par la pyruvate deshydrogénase : le pyruvate est transformé en acétate, éthanol et $\mathrm{CO}_{2}$ par la pyruvate deshydrogénase chez les streptocoques (Broome et al, 1980; Brown et Collins, 1977) préférentiellement en aérobiose, ce système enzymatique comprend : une pyruvate décarboxylase, une lipoate acétyltransférase et une lipoyl deshydrogénase; l'acétyl CoA formé est converti en acétate par l'acétate kinase avec production d'ATP (Dirar et Collins, 1973). Cette activité n'a pas été mise en évidence chez L plantarum;

- par la pyruvate oxydase : une troisième possibilité de métabolisation du pyruvate est celle catalysée par la pyruvate oxydase: chez L plantarum, cette activité est induite par l'oxygène et réprimée par le glucose (Goetz et al, 1980; Murphy et Condon, 1984).

\section{Réactions permettant la réoxydation des pyridines nucléotides: NADH $\rightarrow$ NAD}

Beaucoup des réactions précédentes nécessitent la présence de pyridine nucléotides qui doivent être régénérés. II existe chez les bactéries lactiques 3 types d'oxydases et une peroxydase:

- la NADH oxydase avec production de peroxyde d'hydrogène: cette enzyme a été signalée par Dolin (1955) pour Streptococcus faecalis et sa présence a été confirmée chez beaucoup de bactéries lactiques, mais elle est instable et n'a pas été purifiée;
- la NADH oxydase avec production de $\mathrm{H} 2 \mathrm{O}$ : cette enzyme est répandue chez les bactéries lactiques; elle a été purifiée à partir de L plantarum par Fukui (1961); cette enzyme est inductible par l'oxygène (Murphy et Condon, 1984), mais semble être inactivée à de fortes concentrations d'oxygène (Montville et McFall, 1989) .

- une troisième NADH oxydase, très rare chez les bactéries lactiques, a cependant été observée chez certaines bactéries lactiques cultivées en aérobiose dans un milieu contenant de l'hématine : cette enzyme est associée à la fraction membranaire et peut être considérée comme une chaîne rudimentaire de transport d'électrons réduisant $\mathrm{O}_{2}$ en $\mathrm{H}_{2} \mathrm{O}$ (Pritchard et Wimpenny, 1978).

- une NADH peroxydase : cette enzyme est une flavoprotéine, très répandue chez les bactéries lactiques; elle a été signalée également pour $L$ plantarum ATCC8014 (Goetz et al, 1980); elle réduit le peroxyde d'hydrogène.

Beaucoup de ces activités enzymatiques sont oxygène dépendantes. Des mesures systématiques ont été réalisées dans le cas de $L$ plantarum et de $L$ sake dans les conditions standard de la culture mixte avec $P$ fluorescens.

\section{Dosage des activités enzymatiques}

L'activité de la NADH oxydase a été mise en évidence dans presque tous nos extraits aussi bien pour ceux de $L$ plantarum que de $L$ sake 207 (témoin antagonisme -)

L'activité de la pyruvate oxydase a été mise en évidence dans les extraits de $L$ plantarum. Avec les extraits de $L$ sake 207 aucune activité pyruvate oxydase n'est dosée.

Avec les extraits de $L$ plantarum, la lactate oxydase n'est mise en évidence 
qu'avec addition de cofacteurs : riboflavine, $\mathrm{FAD}, \mathrm{MigCl} 2$. Avec les extraits de $L$ sake 207 , aucune activité lactate oxydase n'a été mise en évidence.

L'activité deshydrogénase NAD indépendante est déterminée par la réduction du DCPIP en présence de lactate par les extraits bactériens. Nous avons pu mesurer dans les extraits de $L$ plantarum CCM 1904 une activité faible. L'accepteur d'électrons in vivo chez $L$ plantarurn n'est pas connu (Murphy et al, 1985).

L'ensemble des dosages enzymatiques faits sur $L$ plantarurn et sur $L$ sake dans nos conditions expérimentales montrent clairement que les potentialités des 2 souches sont très différentes, $L$ sake ne montrant ni activité lactate oxydase ni py- ruvate oxydase. Dans les cultures en conditions anaérobies, ces activités sont absentes ou très faibles chez $L$ plantarum. Ces résultats nous conduisent à proposer un schéma métabolique (fig 4) pour rendre compte de la production d'acétate à partir de lactate chez $L$ plantarum en tenant compte des différences observées avec $L$ sake.

\section{Mise en évidence et régulation de la pyruvate oxydase}

La pyruvate oxydase étant, dans notre hypothèse, une des enzymes clé de la production d'acétate chez $L$ plantarum, nous avons tenté de la mettre en évidence par

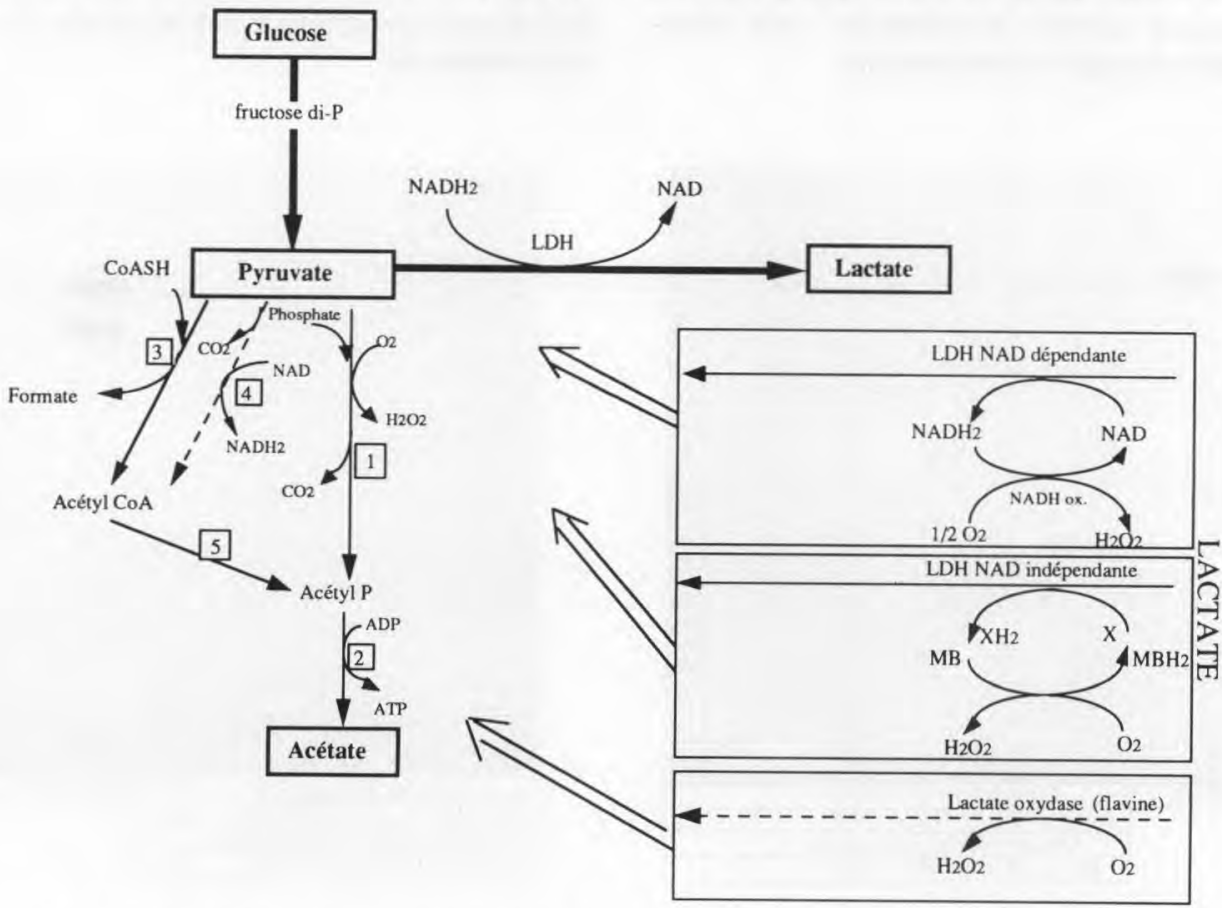

Fig 4. Schéma métabolique chez Lactobacillus plantarum avec variants aboutissant à l'acétate. 1: pyruvate oxydase; 2 : acétate kinase; 3 : pyruvate formate lyase; 4 : pyruvate deshydrogénase; $5:$ phosphate acétyltransférase; LDH : lactate deshydrogénase; MB : bleu de méthylène; $X$ : accepteur d'électrons in vivo non identifié; NADHox: NADH oxydase. 
immunodétection à l'aide d'anticorps antipyruvate oxydase. Ces anticorps ont été obtenus après injection chez le lapin d'une préparation commerciale de pyruvate oxydase de Pediococcus. Grâce à cette détection nous avons pu, dans un deuxième temps, purifier la protéine de $L$ plantarum et obtenir un antiserum contre cette protéine.

Les figures 4 et 5 montrent clairement dans une telle détection la présence dans les extraits de $L$ plantarum de deux bandes protéiques. L'une des bandes n'apparaît qu'après $24 \mathrm{~h}$ de culture, sa présence est corrélée avec une activité pyruvate oxydasique importante. Dans le cas de $L$ sake, une seule bande en position intermédiaire par rapport aux bandes apparaîssant chez $L$ plantarum est visible après $17 \mathrm{~h}$ de culture. Cette bande disparaît par la suite et aucune activité enzymatique n'est décelable dans les mêmes extraits.

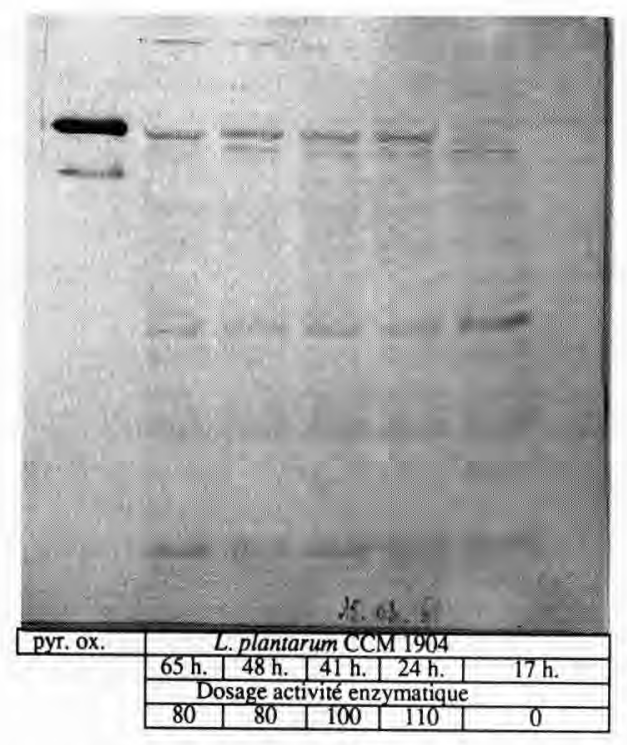

Fig 5. Protéines révélées par les anticorps "anti pyruvate oxydase" et activité de la pyruvate oxydase dans les extraits de $L$ plantarum $\mathrm{CCM}$ 1904 cultivé à $\mathrm{pH} 5,8$ et à $25 \%$ de $\mathrm{pO}_{2}$.

\section{CONCLUSIONS}

Nous avons pu mettre en évidence une activité pyruvate oxydase qui n'apparaît que dans les cellules de $L$ plantarum cultivées en aérobiose. Cette activité semble également être réprimée par le glucose. C'est un des maillons nécessaires à la transformation du lactate, produit dans le métabolisme homofermentaire, en acétate. Cette propriété spécifique de certains lactobacilles est une des possibilités qui permettent d'expliquer l'action inhibitrice de ces souches vis-à-vis de la croissance des pseudomonades en culture mixte. Le clonage du gène de cette enzyme devrait permettre d'approcher les mécanismes de régulation au niveau moléculaire et permettre un éventuel transfert de cette propriété à des souches d'intérêt industriel.

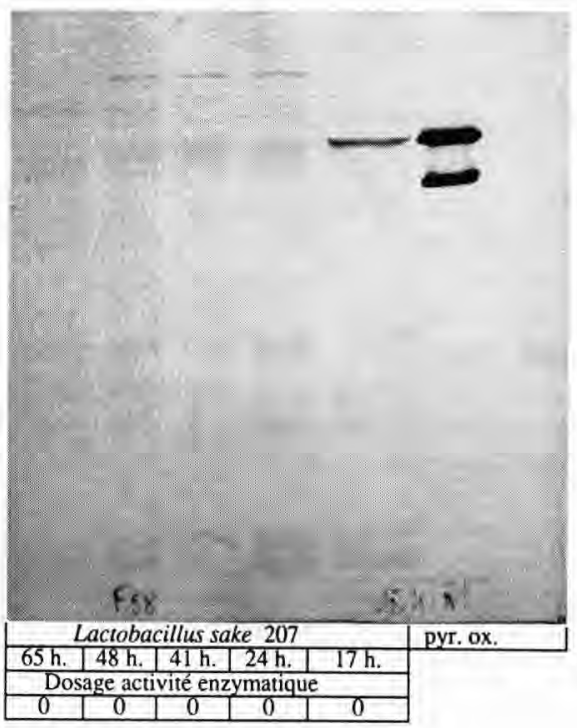

Fig 6. Protéines révélées par les anticorps "antipyruvate oxydase" et activité de la pyruvate oxydase dans les extraits de $L$ sake 207 cultivé à $\mathrm{pH} 5,8$ et à $25 \%$ de $\mathrm{pO}_{2}$. 


\section{RÉFÉRENCES}

Abbe K, Takahashi S, Yamada T (1982) Involvement of oxygen sensitive pyruvate formatelyase in mixed-acid fermentation by Streptococcus mutans under strictly anaerobic conditions. J Bacteriol 152, 175-182

Bernstein IA, Wood HG (1957) Determination of isotopic carbon patterns in carbohydrate by bacterial fermentation. Methods Enzymol 4, 561-584

Broome MC, Thomas MP, Hillier AJ, Jago GR (1980) Pynuvate dehydrogenase activity in group N streptococci. Aust J Biol Sci33,15-25

Brown WV, Collins EB (1977) End-products and fermentation balances for lactic streptococci grown aerobically on low concentrations of glucose. Appl Environ Microbiol 33, 38-42

Crow VL, Pritchard GG (1977) Fructose 1,6diphosphate-activated L-lactate dehydrogenase from Streptococcus lactis : kinetic properties and factors affecting activation. $J$ Bacteriol 131, 82-91

de Man JC, Rogosa M, Sharpe ME (1960) A medium for the cultivation of lactobacilli. $J$ Appl Bacteriol 23, 130-135

de Vries W, Kapteijn WMC, van der Beek EG, Stouthamer AH (1970) Molar growth yields and fermentation balances of Lactobacillus casei L 3 in batch cultures and in continuous cultures. J Gen Microbiol 63, 333-345

Dirar H, Collins EB (1973) Aerobic utilization of low concentration of galactose by Lactobacillus plantarum. J Gen Microbiol 78, 21 1-215

Dolin MI (1955) The DPNH-oxidizing enzymes of Streptococcus faecalis. II. The enzymes utilizing oxygen, cytochrom C, peroxide and 2,6dichlorophenol-indophenol or ferricyanide as oxydants. Arch Biochem Biophys 55, 415-435

Engvall E, Perlmann $P$ (1971) Enzyme-linked immunosorbent assay (ELISA). Quantitative assay of immunoglobulin $\mathrm{G}$. Immunochemistry $8,871-879$

Farber JM, Sanders GW, Dunfield S, Prescott R (1989) The effect of various acidulants on the growth of Listeria monocytogenes. Lett Appl Microbiol 9, 181-183

Fukui S (1961) Crystalline DPNH oxidase from Lactobacillus plantarum. Agric Biol Chem 25, 876-878
Goetz F, Sedewitz B, Elstner EF (1980) Oxygen utilization by Lactobacillus plantarum. I. Oxygen consuming reactions. Arch Microbiol 125, 209-214

Hickey MW, Hillier AJ, Jago GR (1983) Metabolism of pyruvate and citrate in lactobacilli. Aust J Biol Sci 36, 487-496

Ingram M, Ottaway FJH, Coppock JBM (1956) The preservative action of acid substances in food. Chem Ind 1154-1163

Kandler O (1983) Carbohydrate metabolism in lactic acid bacteria. Antonie van Leeuwenhoek J Microbiol Serol 49, 209-224

Lindgren SE, Axelsson LT, Mcfeeters RF (1990) Anaerobic L-lactate degradation by Lactobacillus plantarum. FEMS Microbiol Lett 66, 209-213

Lloyd GT, Hillier AJ, Barlow I, Jago GR (1978) Aerobic formation of acetate from pyruvate by Lactobacillus bulgaricus. Aust $J$ Biol Sci 31, 565-571

Lockridge O, Massey V, Sullivan PA (1972) Mechanism of action of the flavoenzyme lactate oxidase. J Biol Chem 247, 8097-8106

London J (1968) Regulation and function of lactate oxydation in Streptococcus faecium. $J$ Bacteriol 95, 13801387

London J (1990) Uncommon pathways of metabolism among lactic acid bacteria. FEMS Microbiol Rev 87, 103-111

Montel MC, Talon R, Fournaud J, Champomier MC (1991) A simplified key for identifying homofermentative Lactobacillus and Carnobacterium spp from meat. J Appl Bacteriol 70, 469-472

Montville TJ, McFall SM (1989) Oxygen sensitive catabolite distribution in Lactobacillus plantarurn chemostat cultures. Microbios Lett 42, 61-67

Murphy MG, Condon S (1984) Correlation of oxygen utilization and hydrogen peroxide accumulation with oxygen induced enzymes in Lactobacillus plantarurn cultures. Arch Microbiol 138, 44-48

Murphy MG, O'Connor L, Walsh D, Condon S (1985) Oxygen dependent lactate utilization by Lactobacillus plantarum. Arch Microbiol $141,75-79$

Pritchard GG, Wimpenny JWT (1978) Cytochrome formation, oxygen-induced proton extrusion and respiratory activity in Streptococcus faecalis var zymogenes grown in the 
presence of haematin. J Gen Microbiol 104, 15-22

Rhee SK, Pack MY (1980) Effect of environmental pH on fermentation balance of Lactobacillus bulgaricus. J Bacteriol 144, 217-221

Snoswell AM (1966) DL-lactate dehydrogenases (NAD-independent) from Lactobacillus arabinosus. Methods Enzymol 9, 321-327

Strittmatter CF (1959) Electron transport to oxygen in lactobacilli. J Biol Chem 234, 27892793

Sullivan PA (1968) Crystallization and properties of L-lactate oxidase from Mycobacterium smegmatis. Biochem J 110, 363-371
Thomas TD (1987) Acetate production from lactate and citrate by non-starter bacteria in cheddar cheese. N Z J Dairy Sci Technol 22, 25-38

Thomas TD, Ellwood DC, Longyear VMC (1979) Change from homo- to heterolactic fermentation by Streptococcus lactis resulting from glucose limitation in anaerobic chemostat cultures. J Bacteriol 138, 109-117

Tseng CP, Montville TJ (1990) Enzyme activities affecting end product distribution by $L a c$ tobacillus plantarum in response to changes in $\mathrm{pH}$ and $\mathrm{O}_{2}$. Appl Environ Microbiol 56, 2761-2763 